\title{
Topic: Strategic Management of the Epidemiology of Communicable and Non-Communicable Diseases
}

\author{
Article by Morakinyo Ayo Ogunbayode \\ Public Health, Texila American University \\ E-mail:dr_morak@yahoo.com
}

\begin{abstract}
The achievement of creating a healthy environment and life begins with a conscious effort of an individual and then influencing other members of the family, the society and ultimately, the mind-set of the whole country. Efforts from all these parties can help create a conducive environment, free from diseases and comfortable for everybody to live in (Kasenga, 2016).

There was a transition of Millennium Development Goals (MDGs) to Sustainable Development Goals (SDGs) in 2015; this has affected the environment of health plans and strategies. In today's globalized world, rapid urbanization, mechanization of the rural economy, and the activities of trans-national food, drink and tobacco corporations are associated with behavioral changes which include less healthy diet, lower physical activity, tobacco smoking and increased alcohol consumption that increase the risk of chronic non-communicable diseases (NCDs). As a result, population health profiles are rapidly changing. Many in low- and middle-income countries (LMIC) are undergoing rapid changes associated with developing high rates of NCD while concomitantly battling high levels of certain communicable diseases, including HIV, TB and malaria. This has population health, health systems and economic implications for these countries. The rationales of the study are; (a) To highlight the different types of communicable and Non-communicable diseases (b) To highlight different strategic Management of these diseases.
\end{abstract}

Keywords: Communicable diseases, Non-communicable diseases, MDGs, SDGs, LMIC.

\section{Introduction}

The number of deaths and sufferings of human beings due to diseases can be significantly avoided or reduced. This can be achieved by incorporating some routine in our daily living by maintaining a high level of hygiene and sanitation thereby resulting in a healthy lifestyle to avoid some diseases. These routines are affordable, less costly and easy to incorporate (Kasenga, 2016). Hence there should be no excuse from anyone when they acquire any communicable or non-communicable disease. Epidemiology entails the study of the distribution and determinants of health-related states or events (including disease), and the application of this study to the control of diseases and other health problems (http://www.who.int). There are professionals in the field of medicine who look at the causes, modes of transmission and how the conditions can get prevented. It is crucial to come up with strategies on how to manage both communicable and non-communicable diseases. Everyone needs to have the right perception and knowledge of the conditions which affect them and also accepting the responsibility of curbing their occurrences to help minimize deaths caused by diseases. The broad classification of diseases into communicable (infectious) and non-communicable diseases (NCDs) is deeply ingrained. However, this classification may be unhelpful for setting public health priorities, particularly in low- and middleincome countries (LMIC).

\section{Communicable diseases (CDs)}

These are illnesses that are rapidly spread from one person to another; they can also be a spread from an animal to a human being. The spread can occur through a direct contact with contaminated water, feces; infected body fluids e.g. blood urine e.t.c. (Raturi, 2017). Transmission can also be through the air 
like in tuberculosis, examples of Communicable diseases include the common cold, rabies, chicken pox, HIV/AIDS, measles, sore eyes, influenza, and measles. They can easily be prevented through proper hygiene and sanitation, or through vaccination.

\section{Non-communicable diseases (NCDs)}

These are illnesses that cannot be transmitted from one individual to another. NCDs are not caused by infectious agents as in the case of communicable diseases. They are medical conditions that are both noninfectious and non-transmittable. They are chronic in nature that progress slowly and persist for a long time; examples of NCDs are heart diseases, diabetes, kidney failure, hypertension, Alzheimer's disease, various types of cancers and stroke (Raturi, 2017). These conditions need prolonged care management since they develop slowly to levels that might be toxic and dangerous to the body. NCDs can be avoided by educating the society via health talks on a healthy lifestyle.

\section{Strategies for managing communicable and non-communicable diseases Shifting to sustainable development goals (SDGs)}

There was a transition of Millennium Development Goals (MDGs) to SDGs in 2015; this has affected the environment of health plans and strategies. MDGs focused more on health matters which make it relatively straight forward to carry out, three out of the eight goals were health-specific which are MDG 4-6. The fourth MDG focused on reducing the child mortality, and its aim was to reduce the number of children who died by two-thirds from 1990-2015 (Murray, 2015). The fifth MDG focus was aimed at reducing the maternal mortality rate by 75\% from 1990-2015. The MDG 6 aimed at combating HIV/AIDS, malaria and other diseases. The target was relatively simple; to reverse the rapid spread of AIDS and to decrease instances of people getting infected with malaria and other diseases (Nakatani, 2016).

In the SDG only one of the seventeen goals talk about health. The aim of this goal is to ensure healthy lives and promote the well-being of individuals of all ages. It is important to interlink the health sector and health since none is a monopoly of the other (Nakatani, 2016). Good health dramatically contributes greatly to the achievement of different SDG goals and vice versa. The qualities of water and gender bias also have a positive synergy with health. United Nations Development Programme (UNDP) goals in some 170 countries and territories whereby all health strategies are required to apply for the next 15 years, counting from 2016 ( www.UNDP.org ).

\section{Primary prevention}

This refers to activities or a measure, both individual and communal that is directed at reducing the risk of exposure to a risk factor or health determinant in an individual or the population. Some diseases can be avoided by providing vaccination to individuals while they are still young. Governments should ensure all children receive immunizations and at the required age. At birth, a kid should be given vaccines to prevent tuberculosis which is an air-borne disease and polio which is non-communicable. In almost all countries in the world, Polio remains the primary cause of individuals having lives that are disability adjusted (Unwin \& Oni, 2015). Measles, a dangerous communicable illness is also vaccinated against when an infant turns nine months or at six months in the event of a measles outbreak. Vaccines for tetanus and hepatitis B are injected into the body when one is only six weeks of age. Every country should put in place strategies to ensure there is free vaccination in all public hospitals to prevent certain diseases by doing these; the mortality rate will reduce tremendously.

Another example of primary prevention is smoking of tobacco and its risk to cause respiratory diseases Individuals should know the risks and dangers of smoking tobacco through health education. People also know HIV/AIDS is transmitted when they engage in unprotected sex with infected individuals. Primary prevention here will require individuals to take precaution and abstain or use protection anytime they want to have sex with multiple partners. It is common knowledge that sleeping under a treated mosquito 
net helps prevent malaria. Primary prevention goes beyond the scope of the healthcare systems. Everyone needs to put an effort to achieve the standard environmental changes which in turn helps reduce exposure to diseases. (Khasnabis C, Heinicke Motsch K., Achu K., et al., 2010). People need to get sensitized about these factors that would subsequently cause illness.

\section{Secondary prevention}

This focuses on the Sub-clinical stage and the early clinical stage. These measures enable early detection and prompt effective intervention to correct the body from the disease condition to a state of health. Health organizations in collaboration with the governments of various states need to provide free systematic screening programs. A large group of people gets tested and investigated to identify specific persons who face the risk of developing a particular infection (in the case of NCDs), or those who are already infected (Basiro, 2016). This screening could target those people the state considers to be at high risk. In the case of $\mathrm{TB}$ for example, it might focus on the homeless kids and students residing in congested hostels.

The government should ensure there are free services in all public hospitals which provide screening of chronic illnesses like cancer. Detecting these diseases at an early stage will help reduce the deaths caused by cancer. Individuals who show signs of any cancer should start their chemotherapy as soon as they can before symptoms begin to show. Also, equipment used to carry out mammography and cytology screening for breast cancer and cervical cancer respectively need to be easily accessible and set up in all major hospitals. Women should get educated on the early signs of both breast and cervical cancer. They should know how to examine their breasts and detect whether they are reasonable or not. It is advisable to engage a health care provider when deciding on a screening plan that is best for you. Screening should also get provided for other types of cancer like lung cancer, uterine cancer, and prostate cancer. Individuals should be encouraged to control their health by getting regular check-ups and taking cancer screening tests (Komen, 2017).

\section{Good health legislation}

It is up to the government of every country to formulate legislation and policies which will aid in the control and prevention of communicable and non-communicable diseases. There is need to come up with new policies and guidelines on how to manage a communicable and non-communicable disease, It will help reduce the impact of these medical conditions and stop further progression of NCDs. Health-related legislations need to be reviewed on a regular basis to support the control and prevention of these diseases. It is crucial for each government to put regulations on the alcohol and tobacco consumption because excessive alcohol drinking and smoking cigarettes is a risk factor to many diseases. There should be Advocate for all health systems to be sites of awareness on NCDs to the population. Doctors, midwives, nurses, and other health workers should be health promoters to the population on healthy diet, the importance of physical activity and the negative effects of smoking.

\section{Quality medical treatment}

Hospitals should always be ready to treat various illnesses. Treatment is especially necessary when managing communicable diseases like malaria and tuberculosis. Prescribed drugs get given to individuals who are sick by qualified medical professions and at an affordable price. In the case of non-symptomatic illnesses, these infections can be treated early with medicine like isoniazid and a multi-drug regimen in the case of symptomatic infections like TB (Basiro, 2016).

\section{Promotion of physical activity}

Many diseases can be prevented by merely incorporating physical activity and exercises in one's daily routine. Physical activity significantly reduces the risk of developing heart diseases, cancer of the colon, high blood pressure and diabetes among individuals. Governments need to try to implement policies and legislation that promote incorporation of physical activity in one's lifestyle. There should also be public 
awareness of the society by health organizations and medical professionals. Using these platforms, they can discuss the various health benefits of physical activity and how it prevents occurrences of diseases. Physical activity mainly prevents non-communicable diseases like heart diseases, high blood pressure, and obesity. Programs that promote physical activity by members of the society should be implemented in institutions, communities and even at the workplace (Bela \& Mathur, 2011).

\section{Outbreak control}

When there is a suspected case of a disease outbreak, the situation needs to be rapidly investigated and assessed. Typically, these epidemics get indicated after health surveillance teams get put in place. By evaluating the condition in question, the parties involved can decide on what steps to take to control the outbreak. One strategy that can get used in managing the epidemic would be through early detection of the diseases. Once someone is found to be infected, the person gets to receive proper care and effective treatment. The mortality rate will significantly reduce. Also, carrying out prevention activities for the disease in question would be very ideal. Rapid response to an outbreak of a disease is the key to efficiently controlling an emergency (Rayner, 2017). If an individual who has had diarrhea in a particular area is said to have cholera by a doctor, there is a likely hood of more cholera cases in that area, and so prompt measures should be put in place to prevent more individuals from getting affected by cholera. The government must prioritize mapping of communicable diseases outbreaks in human beings.

\section{National public-health surveillance}

It involves the collection of health information, analysis, and dissemination of the same data for appropriate action to be taken. Specific health workers are designated to conduct public-health surveillance. Others need to get placed in temporary relief centers and hospitals. They should be keen to present the list of illnesses these patients have. Active surveillance provides data to help plan on how to intervene in case of an epidemic or an emergency. The inspection can help to provide detailed information on movement patterns on the epidemiology of communicable and non-communicable diseases (Unwin \& Oni, 2015).

\section{Promoting a healthy lifestyle}

Promoting healthy lifestyles among individuals is essential. Interventions need to be implemented to reduce the risk factors for both communicable and non-communicable infections. People get exposed to risk factors of NCDs at a young age though deaths caused by these diseases occur mostly in mature adults. It warrants putting in place appropriate regulatory measures in critical sectors that will be a source of empowerment to individuals and the society as a whole. These governments should have an objective of controlling tobacco intake among smokers and non-smokers, reduction in alcohol abuse and promotion of proper nutritional practices (Rayner, 2017). Such measures could also help to protect children from the adverse effects of partaking unhealthy foods and beverages.

- Proper nutrition: It is essential to eat a balanced and healthy diet to avoid instances of chronic illnesses like diabetes. Governments need to come up with national policies and guidelines to encourage production and consumption of healthy foods.

- Tobacco control: Governments should try and implement initiatives to help tobacco control in school curricula. It is also crucial to mitigate the interference of the tobacco industry in the implementation of public health facilities.

\section{Provisions of a healthy environment}

The control of most communicable infections highly depends on a healthy environment. All governments need to try and prevent infectious diseases amongst individuals by promoting hygiene routine practices. Adequate amounts of clean water should be supplied to members of the community to avoid the occurrence of cholera and diarrhea. These governments also have to ensure that individuals 
have appropriate shelter and adequate sanitation facilities. It merely means that people need to have improved living standards.

Hygiene encompasses various actions undertaken by human beings to maintain a high level of cleanliness of their bodies, their place of work and the domestic environment to prevent the spread of various infections. Health organizations should have public awareness programs where members of the community get taught how to handle domestic animals, wash, prepare their food and even how to dispose of human waste and excretion (Basiro, 2016). These governments should try and provide piped water to domestic households and set up specific sewerages for disposal of waste and polluted water. It is crucial to know that maintenance of personal hygiene helps alleviate suffering such as those caused by bacteria which cause the inflamed skin on people with blocked lymphatic vessels (Basiro, 2016).

Who global action plan for the prevention and control of non-communicable diseases from 2013-2020

1. To raise the priority accorded to the prevention and control of non-communicable diseases in global, regional and national agendas and internationally agreed development goals, through strengthened international cooperation and advocacy

2. To strengthen national capacity, leadership, governance, multisectoral action and partnerships to accelerate country response for the prevention and control of non-communicable diseases

3. To reduce modifiable risk factors for non-communicable diseases and underlying social determinants through creation of health-promoting environments

4. To strengthen and orient health systems to address the prevention and control of non-communicable diseases and the underlying social determinants through people-centered primary health care and universal health coverage

5. To promote and support national capacity for high-quality research and development for the prevention and control of Non-communicable diseases

6. To monitor the trends and determinants of non-communicable diseases and evaluate progress in their prevention and control

\section{Communicable diseases: preparedness, surveillance and response}

Communicable diseases still represent the major public health problems leading to high morbidity and mortality rates among the population, particularly among children under 5 years old. Natural disasters often faced by undeveloped and developing countries turn the population vulnerable to water borne and drought related disease outbreaks such as cholera, dysentery, and meningococcal meningitis.

The major causes of morbidity and mortality are AIDS, malaria, tuberculosis, respiratory infections, diarrhea, and meningitis.

\section{Challenges}

- Weak epidemiological surveillance system lacking infrastructure and appropriate intra and intersectoral coordination;

- Poor health care services, the impact of HIV, and economic migration weakening the health systems;

- Shortage of human resources to implement activities at all levels;

- Lack of radio-communication to liaise remote districts.

\section{Next steps}

- Revitalization/establishment of the epidemic management committees at central, provincial and district levels;

- Establishment and training of Rapid Response Teams on preparedness and response at all levels;

- Adaptation and introduction of the case based and monthly reporting forms in each country's health surveillance system;

- Revision and update the technical guidelines and the training manuals for district health workers; 
DOI: 10.21522/TIJPH.2013.SE.19.01.Art006

ISSN: 2520-3134

- Training for health personnel on disease surveillance, reporting, data management, preparedness and response;

- Sensitization of clinicians at hospitals on disease surveillance with emphasis on cases detection and reporting on priority diseases;

- Development of a comprehensive national laboratory policy;

- Training at provincial labs in meningitis surveillance and provide adequate supplies.

- [WHO/ Communicable diseases: preparedness, surveillance and response (n.d)]

\section{Challenges and limitations of implementing general health}

\section{Strategies and plans}

- Inadequate and unstable sources of funding for health care providers.

- Poor living standards in most third world countries. It leads to contraction of communicable diseases like diarrhoea, cholera, tuberculosis, and malaria since most of these people do not have ways to prevent these infections.

- Aging: A majority of the world's population today are individuals aged sixty-five years and above. These individuals end up getting various medical conditions and impairments that impede longevity.

- The existence of a health system which regards neoliberal principles as the driver of its actions.

- Poor health policies implementation by the government. The moment the government does not prioritize health matters, then a significant crisis is likely to occur. Policymakers must come up with guidelines and policies to get implemented for better provision of health care services in various countries.

- There is limited or no involvement of institutions outside the health sector in generating responses that link various areas as well as organizations that promote community involvement and participation in issues affecting the health sector.

- Lack of competent health workers who can engage in community orientation or focus on the health status of the family. Most of these workers rather just treat patients whenever they fall ill or are involved in accidents.

\section{Summary}

Now, at the dawn of the third millennium, non-communicable diseases are sweeping the entire globe. There is an increasing trend in developing countries, where the demographic and socio-economic transition imposes more constraints on dealing with the double burden of infectious and non-infectious diseases in a poor environment, characterized by ill-health systems. It is predicted that, by 2020, noncommunicable diseases will cause seven out of every ten deaths in developing countries. Among noncommunicable diseases, special attention is devoted to cardiovascular disease, diabetes, cancer and chronic pulmonary disease. The burden of these conditions affects countries worldwide but with a growing trend in developing countries. Preventative strategies must take into account the growing trend of risk factors correlated to these diseases. In parallel, despite the success of vaccination programmes for polio and some childhood diseases, other diseases like AIDS, tuberculosis, malaria and dengue are still out of control in many regions of the globe. This paper is a brief review of recent literature dealing with communicable and non-communicable diseases in developing countries. It gives a global view of the main diseases and their impact on populations living in low- and middle-income nations. [Abdesslam Boutayeb; 1 March 2006].

\section{Conclusion}

There is the need for urgent attention and extensive research looking at the magnitude of the epidemiology of both communicable and non-communicable diseases. There have been enormous efforts in the past to attempt and eradicate the occurrence of these illnesses by applying MDGs and from 2016, SDGs. However, this issue still needs to be looked into and addressed in an entirely different manner. The 
DOI: 10.21522/TIJPH.2013.SE.19.01.Art006

ISSN: 2520-3134

epidemiology of the various communicable and non-communicable diseases has proven to be the leading cause of mortality worldwide. The case gets even worse for the low-income regions. Though it is common knowledge that proper hygiene, sanitation, improved standards of living and leading healthy lifestyles reduce occurrences of these diseases, there still are barriers to ensuring adequate safety measures get adhered to by all persons. Governments and other health organizations need to come up with strategies to deal with this situation. In this way, the mortality rates will get significantly reduced. People who are healthy are of good economic importance to their respective countries.

\section{Acknowledgements}

I would like to express my sincere gratitude to my Student coordinators so far in my program -Ms Suma Menon, Ms Ramyaa for their relentless support and assistance given to me through of the period of the program. I also want to appreciate Ms Jasmin Hanley for her guide to write my dissertation.

I thank Dr. Afortu-Ofre Mathias Ofre for all his assistance during my program. A special appreciation to my beloved wife, Shayreemer L. Ogunbayode for her endless support and understanding from the beginning of the program to the very end of it, words are not enough to describe my heart gratitude to everyone that have contributed to the success of my Master's program, Above all I want to give all thanks to God almighty for his strength and guidance through it all.

\section{References}

[1].Abdesslam Boutayeb; (1 March 2006, Pages 191-199) The double burden of communicable and noncommunicable diseases in developing countries, Transactions of The Royal Society of Tropical Medicine and Hygiene, Volume 100, Issue 3, Retrieved from: https://doi.org/10.1016/j.trstmh.2005.07.021.

[2].Basiro, D. 9 2016). Public Health Approaches to Infectious diseases. The Open University. Retrieved from: http://www.open.edu/openlearn/ocw/mod/oucontent/view.php?id=8537\&printable=1.

[3].Bela, S. \& Mathur, P. (2011). Research Priorities for Prevention and Control of Non-Communicable Diseases in India. Indian Journal of Community Medicine, 36 (Supp11); s72-s77.

[4].Communicable diseases: preparedness, surveillance and response (n.d) Retrieved from: http://www.who.int/countries/moz/areas/communicable_diseases/en/index2.html.

[5].Kasenga, F. H. (2016). Epidemiology of Communicable and Non-Communicable Diseases_Attributes of Lifestyle and Nature on Humankind. Intech.

[6].Khasnabis C, Heinicke Motsch K., Achu K. et al. (2010). Community-Based Rehabilitation: CBR Guidelines. Geneva: World Health Organization Prevention. Retrieved from https://www.ncbi.nlm.nih.gov/books/NBK310943/

[7].Komen, S. G. (2017). The Best way to improve your chances for Survival. Murray C. (2015). Shifting to Sustainable Development Goals-Implications for Global Health. $N$ Eng J Med. pp.1390-3. doi 10.1056/NEJMp1510082.

[8].Nakatani, H. (2016). Global Strategies for the Prevention and Control of Infectious Diseases and NonCommunicable Diseases. Journal of Epidemiology. Elsevier Publishers; 26(4), pp. 171-178. doi: 10.2188/jea.JE201600100.

[9].Raturi, S. (2017). What are Communicable and Non-Communicable Diseases? Quora. Panjab University, Swami Sarvanand. Retrieved from https://www.quora.com/What-are-communicable-and-non-communicable diseases?

[10]. Rayner, M. (2017). Non-Communicable Diseases Prevention Strategies. University of Oxford.

[11]. Unwin, N. \& Oni, T. (2015). Why the Communicable and Non-Communicable Diseases Dichotomy is Problematic for the Public Health Control Strategies in an era of Health Transition. Retrieved from International Health, Oxford Journals; 7(6), pp.390-399. Doi 10.10.93/inthealth/ihv040.

[12]. WHO/ Global Action Plan for the Prevention and Control of Non-communicable diseases from 2013-2020

Retrieved from: http://www.who.int/nmh/publications/ncd-action-plan/en/. 